\title{
The Two-User Cutoff Rate for an Asynchronous and a Synchronous Multiple-Access Channel Are the Same
}

\author{
PRAKASH NARAYAN, STUDENT MEMBER, IEEE, AND DONALD L. SNYDER, FELLOW, IEEE
}

\begin{abstract}
The cutoff rate region for block coding of a two-sender one-receiver multiple-access channel is shown to be the same with and without frame synchronization between the two senders.
\end{abstract}

\section{INTRODUCTION}

$\mathrm{C}$ OVER, MCELIECE, AND POSNER [1] have shown in a recent report that for block coding, the capacity region for a discrete memoryless two-user multiple-access channel with one receiver does not depend on whether the two users have frame synchronization. Our objective is to establish for their model that the same is true for the cutoff rate region. This is important because the cutoff rate is gencrally more readily achieved practically than is capacity. Also, at least for single user communication, the cutoff rate parameter $R_{0}$ has proven to be convenient and tractable in design considerations.

The use of the cutoff rate parameter $R_{0}$ in the study of single user coded communication systems was first advocated by Wozencraft and Kennedy [2] in 1966. In 1974, Massey [3] gave an eloquent argument in favor of $R_{0}$ as a criterion for the coordinated design of the modulation and coding in a communication system. By interpreting $R_{0}$ as a function of the modulator and demodulator, it became evident how $R_{0}$ could be used to design the best discrete channel seen by the encoder and decoder.

For a single user system with block coding, the cutoff rate parameter is obtained by maximizing the negative exponent in the expression for the upper bound on the average error probability as a function of a free parameter $\rho$ in order to obtain the tightest upper bound [4, p. 142]. For a block encoder of rate $R$ and codeword length $n$, the probability of decoding error is bounded above according to $P_{e} \leq \exp \left[-n\left(R_{0}-R\right)\right]$ for all rates $R$ less than or equal to $R_{0}$. Similarly, for convolutional coding with maximum likelihood decoding, Viterbi [5] has shown that the probability of error is upper bounded according to $P_{e} \leq$

Manuscript received December 1, 1979; revised June 30, 1980. This work was supported by the National Science Foundation under Grant ENG 76-11565 and by the National Institute of Health under Grant RR00396 from the Division of Research Resources.

The authors are with the Department of Electrical Engineering, Washington University, P.O. Box 1127, St. Louis, MO 63130.
$k_{R} \exp \left(-n R_{0}\right)$ for all rates $R$ less than or equal to $R_{0}$, where $n$ is the constraint length, $R$ is the code rate, and $k_{R}$ is a weakly dependent function of $R$. Also, $R_{0}$ is the " $R_{\text {comp }}$ " of sequential decoding; that is, $R_{0}$ is the rate above which the average number of decoding steps per decoded digit becomes unbounded. It is evident from these results, and has been noted by others, that for both block and convolutional coding $R_{0}$ determines a range of rates over which reliable communication can be assured as well as the coding complexity needed to achieve a specified level of reliability. Thus as a single parameter characterizing a channel, $R_{0}$ is more informative than the more fundamental channel capacity because the capacity indicates nothing about complexity even though it does give the entire range of rates over which any degree of reliability can be achieved.

Compared to other single parameters that might be adopted to characterize a channel, such as $R_{x}, R_{\text {crit }}$, and $R_{1}$ [2, 4 (p. 160)], the cutoff rate $R_{0}$ has several advantages. It is more tractable than the others, especially in the context of optimal modulator design. Also, since $R_{0}$ is larger than the other three parameters, it provides a better estimate of the capacity and a tighter upper bound on the error probability and, therefore, yields a less conservative estimate of the complexity needed to achieve a specified reliability.

The practical implications of the $R_{0}$ parameter for single user channels were first demonstrated by Massey [3] who used it to identify optimal modulator designs for an additive white Gaussian noise channel. Snyder and Rhodes [6] used similar ideas for direct detection optical communication systems. Lee [7] used the $R_{0}$ parameter to obtain necessary conditions for the optimal coherent demodulation of $M$-ary signals on an additive white Gaussian channel. These studies and several others [8]-[10] suggest that the cutoff rate is a very useful parameter characterizing a single user channel.

We have generalized the cutoff rate parameter for a single user channel to a "cutoff rate region" for a multiple access channel. Our preliminary studies indicate that the cutoff rate region is likewise a tractable parameter useful in design considerations. As an example, for any rate pair in 
the cutoff rate region, we shall estimate the additional encoding/decoding complexity required without frame synchronization to assure the same reliability as with synchronization. Issues associated with the coordinated design of encoders and modulators for multiple access channels will be explored using the cutoff rate region in another paper.

A two-user multiple access channel is specified by a set of transition probabilities $p(w \mid u, v)$, where $w$ is a received letter in the alphabet $Y$, and $u$ and $v$ are codeletters sent by sender- 1 and sender-2, respectively, from their alphabets $X_{1}$ and $X_{2}$. Codewords are assumed to be selected independently by the two senders so that if $Q_{1}\left(x_{1 i}\right)$ is the probability that sender-1 chooses his $i$ th codeword, and if $Q_{2}\left(x_{2 j}\right)$ is the probability that sender- 2 chooses his $j$ th codeword, then the joint probability $Q\left(x_{1 i}, x_{2 j}\right)$ that sender-1 and sender-2 choose these codewords satisfies

$$
Q\left(x_{1 i}, x_{2 j}\right)=Q_{1}\left(x_{1 i}\right) Q_{2}\left(x_{2 j}\right),
$$

for all $i, j$.

We assume that the channel is memoryless and that codewords of length $n$ are constructed by independently selecting $n$ codeletters according to a common distribution $q_{1}$ for sender- 1 and $q_{2}$ for sender- 2 . Then

$$
\begin{aligned}
P\left(y \mid x_{1}, x_{2}\right) & =\prod_{m=1}^{n} p\left(y_{m} \mid x_{1 m}, x_{2 m}\right), \\
Q_{1}\left(x_{1}\right) & =\prod_{m=1}^{n} q_{1}\left(x_{1 m}\right), \\
Q_{2}\left(x_{2}\right) & =\prod_{m=1}^{n} q_{2}\left(x_{2 m}\right),
\end{aligned}
$$

and

$$
\begin{aligned}
Q\left(x_{1}, x_{2}\right) & =\prod_{m=1}^{n} q\left(x_{1 m}, x_{2 m}\right) \\
& =\prod_{m=1}^{n} q_{1}\left(x_{1 m}\right) q_{2}\left(x_{2 m}\right) .
\end{aligned}
$$

Slepian and Wolf [11] have demonstrated for a synchronous discrete memoryless two-sender one-receiver multipleaccess channel that there exists a block code of codeword length $n$ and a rate pair $\left(R_{1}, R_{2}\right)$ such that the average probability of decoding error, for maximum likelihood decoding, satisfies

$$
\bar{P}_{e, \text { sync }} \leq \sum_{\alpha=1}^{3} \exp \left\{-n\left[-\rho_{\alpha} R_{\alpha}+E_{\alpha}\left(\rho_{\alpha}, q, p\right)\right]\right\}
$$

for all $\rho_{\alpha}$ between or equal to 0 and 1 , where

$$
\begin{aligned}
R_{\alpha} & =R_{1}, & & \alpha=1, \\
& =R_{2}, & & \alpha=2, \\
& =R_{1}+R_{2}, & & \alpha=3,
\end{aligned}
$$

and where

$$
\begin{aligned}
& E_{1}\left(\rho_{1}, q, p\right) \\
& \quad=-\ln \left(\sum_{Y} \sum_{X_{2}} q_{2}(v)\left[\sum_{X_{1}} q_{1}(u) p(w \mid u, v)^{\left(1+\rho_{1}\right)^{-1}}\right]^{\left(1+\rho_{1}\right)}\right),
\end{aligned}
$$

$$
\begin{aligned}
& E_{2}\left(\rho_{2}, q, p\right) \\
& =-\ln \left(\sum_{Y} \sum_{X_{1}} q_{1}(u)\left[\sum_{X_{2}} q_{2}(v) p(w \mid u, v)^{\left(1+\rho_{2}\right)^{-1}}\right]^{\left(1+\rho_{2}\right)}\right),
\end{aligned}
$$

and

$E_{3}\left(\rho_{3}, q, p\right)$

$$
=-\ln \left(\sum_{Y}\left[\sum_{X_{1}} \sum_{X_{2}} q(u, v) p(w \mid u, v)^{\left(1+\rho_{3}\right)^{-1}}\right]^{\left(1+\rho_{3}\right)}\right) .
$$

In order to identify the cutoff rate region for a synchronous two-user multiple-access channel, we minimize the bound in (1) as a function of $\rho_{\alpha}$ for $\alpha=1,2,3$. This minimization can be effected by minimizing each exponential term separately using Gallager's method [6, p. 142] and occurs at $\rho_{1}=\rho_{2}=\rho_{3}=1$. The cutoff rate region is then the convex hull of the union, over all independent probability distributions $q(u, v)=q_{1}(u) q_{2}(v)$, of the set of all rate pairs $\left(R_{1}, R_{2}\right)$ that satisfy

$$
\begin{aligned}
& R_{1} \leq-\ln \left(\sum_{Y} \sum_{X_{2}} q_{2}(v)\left[\sum_{X_{1}} q_{1}(u) p^{1 / 2}(w \mid u, v)\right]^{2}\right) \\
& R_{2} \leq-\ln \left(\sum_{Y} \sum_{X_{1}} q_{1}(u)\left[\sum_{X_{2}} q_{2}(v) p^{1 / 2}(w \mid u, v)\right]^{2}\right),
\end{aligned}
$$

and

$$
R_{1}+R_{2} \leq-\ln \left(\sum_{Y}\left[\sum_{X_{1}} \sum_{X_{2}} q(u, v) p^{1 / 2}(w \mid u, v)\right]^{2}\right) .
$$

With frame synchronization, time sharing achieves any rate pair $\left(R_{1}, R_{2}\right)$ in the convex hull. It will be demonstrated that this cutoff rate region remains the same without frame synchronization between the two users, provided a finite upper bound " $d$ " on the time shifts between them is known.

\section{Review of THE COVER-McEliece-PosNer Model}

We adopt the following definitions due to Cover, McEliece, and Posner [1].

Definition 1: The relative maximum delay $d$ is the maximum amount by which a message from sender-1 and a message from sender- 2 can be out of synchronism relative to a universally known time.

Definition 2: An $\left\{\left(M_{1}, M_{2}\right), n, d, P_{n}\right\}$ code for a twouser multiple access channel with a relative maximum delay $d$ is a pair of encoding maps $x_{1}:\left\{1,2, \cdots, M_{1}\right\} \rightarrow X_{1}^{n}$ and $x_{2}:\left\{1,2, \cdots, M_{2}\right\} \rightarrow X_{2}^{n}$, and a decoding map $g: Y^{n} \rightarrow$ $\left\{1,2, \cdots, M_{1}\right\} \times\left\{1,2, \cdots, M_{2}\right\}$, where $X_{i}$ is the code alphabet of sender- $i, M_{i}$ is the next integer greater or equal to $\exp \left(n R_{i}\right)$, and $R_{i}$ is the rate of sender- $i, i=1,2 . Y$ is the output alphabet.

Definition 3: For any given maximum relative delay $d$, a pair of rates $\left(R_{1}, R_{2}\right)$ will be said to be achievable if there exists a sequence of $\left\{\left(M_{1}, M_{2}\right), n, d, P_{n}\right\}$ codes such that $P_{n}$ tends to zero as as $n$ tends to infinity. 
Example 1 (Binary Adder Channel): Let $X_{1}=X_{2}=\{0,1\}$ and $y=x_{1}+x_{2}$. Then, $Y=\{0,1,2\}$. The output symbol $y=1$ is an erasure. Cover, McEliece, and Posner [1] and Gaarder and Wolf [12] proved the capacity region for this two-sender binary-adder multiple-access channel to be $R_{1}$ $\leq 1, R_{2} \leq 1, R_{1}+R_{2} \leq 1.5$ bits. Using (2)-(4), we find the cutoff rate region to be $R_{1} \leq 1, R_{2} \leq 1, R_{1}+R_{2} \leq 1.42$ bits.

For an asynchronous multiple-access channel, the cutoff rate region may not be convex. Then we must find a convex combination of rate pairs $\left(R_{1}, R_{2}\right)$ and $\left(R_{1}^{\prime}, R_{2}^{\prime}\right)$ to achieve the pair $\left(R_{1}^{*}, R_{2}^{*}\right)=\beta\left(R_{1}, R_{2}\right)+(1-\beta)\left(R_{1}^{\prime}, R_{2}^{\prime}\right)$ for arbitrary $\beta, 0 \leq \beta \leq 1$. This will be accomplished in the remainder of the paper as follows. First we let $Q\left(x_{1}, x_{2}\right)$ induce a cutoff-rate region that has $\left(R_{1}, R_{2}\right)$ as an extreme point. In the same way, we let $Q^{\prime}\left(x_{1}, x_{2}\right)$ induce a cutoffrate region that has $\left(R_{1}^{\prime}, R_{2}^{\prime}\right)$ as an extreme point. Using a random coding procedure, generate a random $\left\{\left(e^{\beta n R_{1}}, e^{\beta n R_{2}}\right), \beta n\right\}$ code with codewords $x$ of length $\beta n$ and codeword probability $Q$ and a random $\left\{\left(e^{(1-\beta) n R_{1}^{\prime}}, e^{(1-\beta) n R_{2}^{\prime}}\right),(1-\beta) n\right\}$ code with codewords $x^{\prime}$ of length $(1-\beta) n$ and codeword probability $Q^{\prime}$. The transmitted codewords are obtained by the concatenation $x x^{\prime}$ and have length $n$.

Corresponding to the message pair $(i, j)$, sender- 1 transmits codewords $x_{1 i} x_{1 i}^{\prime}$, and sender- 2 transmits codeword $x_{2 j} x_{2 j}^{\prime}$. Assume that messages transmitted by each sender are selected with equal probability, so $(i, j)$ is transmitted with probability $\left(M_{1} M_{2}\right)^{-1}$. The receiver observes $y$ and searches for $x_{1} x_{1}^{\prime}$ and $x_{2} x_{2}^{\prime}$ with arbitrary time shifts up to a maximum relative delay $d$. Because of the lack of frame synchronization, up to $d$ of the leading symbols received in a particular transmission interval may contain interference from symbols of previous transmission intervals and must be ignored.

For some $k_{1}$ and $k_{2}$ between or equal to 0 and $d$, the transmitted and received sequences will have the appearance indicated in Fig. 1. The important feature implied in this figure is that $x_{1} x_{1}^{\prime}$ and $x_{2} x_{2}^{\prime}$ will have a sufficient overlap to enable decoding with an arbitrarily small error probability. The regions of overlap can be preidentified and are at least of lengths $\beta n-d$ and $(1-\beta) n-d$, independent of $k_{1}$ and $k_{2}$.

Let $T^{k}(v)$ denote a right shift by $k$ symbols of an $n$-tuple $v=\left(v_{1}, v_{2}, \cdots, v_{n}\right)$. Also, let $W(v)$ denote the window function that extracts the $\beta n-d$ symbols of the $n$-tuple $v$ appearing in the first window in Fig. 1 . Then $W(v)=\left(v_{d+1}, v_{d+2}, \cdots, v_{\beta n}\right)$ and $W\left[T^{k}(v)\right]=$ $\left(v_{k+d+1}, v_{k+d+2}, \cdots, v_{k+\beta n}\right)$.

The decoding algorithm decodes a received $n$-tuple $y$ as the message pair $\left(i^{*}, j^{*}\right)$ that maximizes the function $f(i, j)$ defined by

$$
\begin{array}{r}
f(i, j)=\max _{1 \leq k_{1}, k_{2} \leq d} P\left(W(y) \mid W\left[T^{k_{1}}\left(x_{1 i} x_{1 i}^{\prime}\right)\right],\right. \\
\left.W\left[T^{k_{2}}\left(x_{2 j} x_{2 j}^{\prime}\right)\right]\right)
\end{array}
$$

for all $(i, j) \in\left\{1,2, \cdots, M_{1}\right\} \times\left\{1,2, \cdots, M_{2}\right\}$.
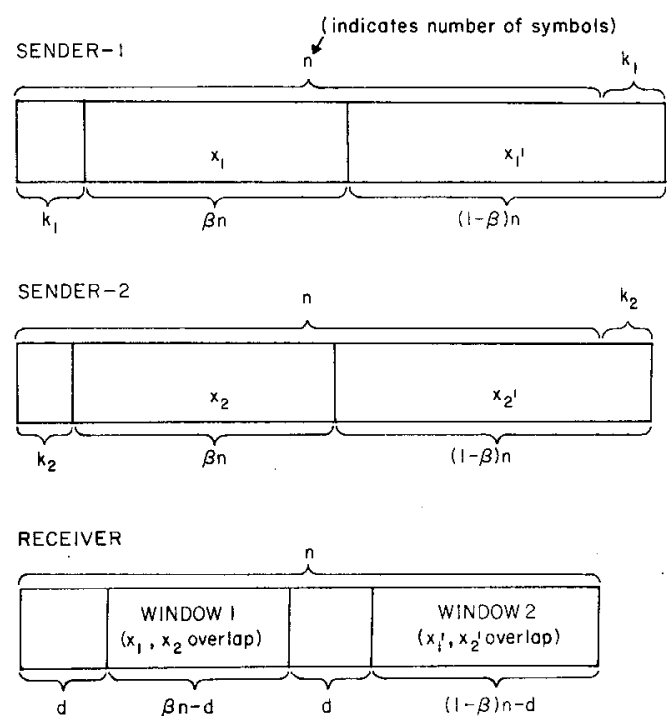

Fig. 1. Asynchronous sender codewords and their overlap windows in the received word.

\section{Cutoff Rate Region without Frame SYNCHRONIZATION}

Assuming that message $(i, j)$ is transmitted and that $y$ is decoded as $\left(i^{*}, j^{*}\right)$, a decoding error can occur in the following three ways: $\left(i^{*} \neq i, j^{*}=j\right),\left(i^{*}=i, j^{*} \neq j\right)$, and $\left(i^{*} \neq i, j^{*} \neq j\right)$. Denote the probability for each of these errors by

$$
\begin{aligned}
& P_{1}=\operatorname{Pr}\left(i^{*} \neq i, j^{*}=j\right), \\
& P_{2}=\operatorname{Pr}\left(i^{*}=i, j^{*} \neq j\right), \\
& P_{3}=\operatorname{Pr}\left(i^{*} \neq i, j^{*} \neq j\right) .
\end{aligned}
$$

We initially upper bound $P_{1}$ over the ensemble of codes considering only the first window in Fig. 1 . Let $P_{1,1}$ be the probability of event $\left(i^{*} \neq i, j^{*}=j\right)$ considering window-1 alone, over the ensemble of codes. The following upper bound on $P_{1,1}$ can be obtained using Gallager's method [6, pp. 135-138]:

$$
\begin{aligned}
P_{1,1} \leq & \sum_{k_{1}, k_{2}=1}^{d}\left[\sum_{W\left(x_{2 j} x_{2 j}^{\prime}\right)} Q_{2}\left\{W\left[T^{k_{2}}\left(x_{2 j} x_{2 j}^{\prime}\right)\right]\right\}\right. \\
& \cdot \sum_{W(y)} \sum_{W\left(x_{1 i} x_{1 i}^{\prime}\right)} Q_{1}\left\{W\left[T^{k_{1}}\left(x_{1 i} x_{1 i}^{\prime}\right)\right]\right\} \\
& \cdot P\left(W(y) \mid W\left[T^{k_{1}}\left(x_{1 i} x_{1 i}^{\prime}\right)\right], W\left[T^{k_{2}}\left(x_{2 j} x_{2 j}^{\prime}\right)\right]\right) \\
& \cdot \operatorname{Pr}\left(i^{*} \neq i \mid i, W\left[T^{k_{1}}\left(x_{1 i} x_{1 i}^{\prime}\right),\right.\right. \\
& \left.\left.\cdot W\left[T^{k_{2}}\left(x_{2 j} x_{2 j}^{\prime}\right)\right], W(y)\right)\right] .
\end{aligned}
$$

The last factor in (8) is the probability of a decoding error only in the message transmitted by sender- 1 conditioned on the message $i$ of sender-1, the appearance in the first window of the particular portion of the $i$ th codeword of sender- $i$ after a shift of $k_{1}$ symbols due to asynchronism, the appearance in the first window of the particular por- 
tion of the $j$ th codeword of sender-2 after a shift of $k_{2}$ symbols due to asynchronism, and the portion of $y$ in the first window. Here the message $j$ of sender- 2 is fixed, and no error is made in its decoding.

For a given $k_{1}, k_{2}, i$, and $j$, we have $i^{*} \neq i$ if

$$
L\left(i^{*}, i\right) \geq 1,
$$

where $L\left(i^{*}, i\right)$ is the likelihood ratio

$L\left(i^{*}, i\right)=\frac{P\left(W(y) \mid W\left[T^{k_{1}}\left(x_{1 i^{*}} x_{1 i^{*}}^{\prime}\right)\right], W\left[T^{k_{2}}\left(x_{2 j} x_{2 j}^{\prime}\right)\right]\right)}{P\left(W(y) \mid W\left[T^{k_{1}}\left(x_{1 i} x_{1 i}^{\prime}\right)\right], W\left[T^{k_{2}}\left(x_{2 j} x_{2 j}^{\prime}\right)\right]\right)}$.

Let $\Omega$ denote the collection of windowed codewords $\left\{W\left(x_{1 i^{*}} x_{1 i^{*}}^{\prime}\right)\right\}$. Also, for a given $i$, let $\Omega_{L}\left(k_{1}, i\right)$ be the subset of $\Omega$ for which $L\left(i^{*}, i\right) \geq 1$. Define $A\left(k_{1}, i^{*}\right)$ as the event that the windowed codeword $\left(W\left(x_{1 i^{*}} x_{1 i^{*}}^{\prime}\right)\right)$ is in $\Omega_{L^{\prime}}$. Then

$$
\begin{array}{r}
\operatorname{Pr}\left(i^{*} \neq i \mid i, W\left[T^{k_{1}}\left(x_{1 i^{*}} x_{1 i^{*}}^{\prime}\right)\right], W\left[T^{k_{2}}\left(x_{2 j} x_{2 j}^{\prime}\right)\right], W(y)\right) \\
\leq \operatorname{Pr}\left[\bigcup_{i^{*} \neq i} A\left(k_{1}, i^{*}\right)\right] \leq\left[\sum_{i^{*} \neq i} \operatorname{Pr}\left\{A\left(k_{1}, i^{*}\right)\right\}\right]^{\rho_{1}}
\end{array}
$$

for all $\rho_{1}$ between or equal to zero and one.

We now bound the probability of event $A\left(k_{1}, i^{*}\right)$ according to

$$
\begin{aligned}
\operatorname{Pr}\left[A\left(k_{1}, i^{*}\right)\right] & =\sum_{\Omega_{L}} Q\left[W\left(x_{1 i^{*}} x_{1 i^{*}}^{\prime}\right), W\left(x_{2 j} x_{2 j}^{\prime}\right)\right] \\
& \leq \sum_{\Omega} Q\left[W\left(x_{1 i^{*}} x_{1 i^{*}}^{\prime}\right), W\left(x_{2 j} x_{2 j}^{\prime}\right)\right] L^{s}\left(i^{*}, i\right)
\end{aligned}
$$

for all $s \geq 0$.

Using (11) in (10) and defining $M_{W}=\exp \left[(\beta n-d) R_{1}\right]$ as the number of distinct codeletter sequences that can appear in window-1 due to sender-1, we obtain

$$
\begin{aligned}
& \operatorname{Pr}\left(i^{*} \neq i \mid i, W\left[T^{k_{1}}\left(x_{1 i} x_{1 i}^{\prime}\right)\right], W\left[T^{k_{2}}\left(x_{2 j} x_{2 j}^{\prime}\right)\right], W(y)\right) \\
& \leq\left[\left(M_{W}-1\right) \sum_{\Omega} Q\left[W\left(x_{1 i^{*}} x_{1 i^{*}}^{\prime}\right), W\left(x_{2 j} x_{2 j}^{\prime}\right)\right] L^{s}\left(i^{*}, i\right)\right]^{\rho_{1}} .
\end{aligned}
$$

Substituting (12) into (8) and interchanging the order of summation, we obtain
Using the definition of $L\left(i^{*}, i\right)$, setting $s=\left(1+\rho_{1}\right)^{-1}$, and recognizing that $Q_{2} \leq 1$, we obtain

$$
\begin{aligned}
P_{1,1} \leq & \left(M_{W}-1\right)^{\rho_{1}} \sum_{k_{1}, k_{2}=1}^{d}\left[\sum_{W\left(x_{2 j^{\prime}} x_{j}^{\prime}\right)} Q_{2}\left\{W\left[T^{k_{2}}\left(x_{2 j} x_{2 j}^{\prime}\right)\right]\right\}\right. \\
& \cdot \sum_{W(y)}\left\{\sum_{\Omega} Q_{1}\left\{W\left[T^{k_{1}}\left(x_{1 i} x_{1 i}^{\prime}\right)\right]\right\}\right. \\
& \cdot\left\{P \left(W(y) \mid W\left[T^{k_{1}}\left(x_{1 i^{*}} x_{1 i^{*}}^{\prime}\right)\right]\right.\right. \\
& \left.\left.\left.\left.W\left[T^{k_{2}}\left(x_{2 j^{\prime}} x_{2 j}^{\prime}\right)\right]\right)\right\}^{\left(1+\rho_{1}\right)^{-1}}\right\}^{1+\rho_{1}}\right] .
\end{aligned}
$$

Using the assumed memoryless and independence properties of the channel and code selection, and recognizing that $x_{1 i} x_{1 i}^{\prime}$ and $x_{2 j} x_{2 j}^{\prime}$ are dummy summation variables, we then obtain

$$
\begin{aligned}
P_{1,1} & \leq \sum_{k_{1}, k_{2}=1}^{d} \exp \left\{-(\beta n-d)\left[E_{1}\left(\rho_{1}, q, P\right)-\rho_{1} R_{1}\right]\right\} \\
& \leq d^{2} \exp \left\{-(\beta n-d)\left[E_{1}\left(\rho_{1}, q, P\right)-\rho_{1} R_{1}\right]\right\},
\end{aligned}
$$

where we define

$$
\begin{aligned}
& E_{1}\left(\rho_{1}, q, p\right) \\
& \quad=-\ln \left(\sum_{Y} \sum_{X_{2}} q_{2}(v)\left[\sum_{X_{1}} q_{1}(u) p(w \mid u, v)^{\left(1+\rho_{1}\right)^{-1}}\right]^{1+\rho_{1}}\right) .
\end{aligned}
$$

Hence,

$$
P_{1,1} \leq \exp \left\{-(\beta n-d)\left[E_{1}\left(\rho_{1}, q, p\right)-\rho_{1} R_{1}-\frac{2 \ln (d)}{\beta n-d}\right]\right\} .
$$

Since all messages are equiprobable, $P_{1,1}$ is also the average probability of error $\bar{P}_{1,1}$ in decoding the first sender's message inspecting window- 1 alone. By inspecting window- 2 alone, we similarly find the average probability of error, $\bar{P}_{1,2}$, in decoding the first sender's message as

$$
\begin{aligned}
\bar{P}_{1,2} \leq \exp \{ & -[(1-\beta) n-d] \\
& \left.\cdot\left[E_{1}\left(\rho_{1}^{\prime}, q, p\right)-\rho_{1}^{\prime} R_{1}^{\prime}-\frac{2 \ln (d)}{(1-\beta) n-d}\right]\right\},
\end{aligned}
$$

where $0 \leq \rho_{1}{ }^{\prime} \leq 1$. Thus, the average probability of error $\bar{P}_{1}$ in decoding the first sender's message incorrectly while

$$
\begin{aligned}
P_{1,1} \leq & \left(M_{W}-1\right)^{\rho_{1}} \sum_{k_{1}, k_{2}=1}^{d}\left[\sum _ { W ( x _ { 2 j } x _ { 2 j } ^ { \prime } ) } Q _ { 2 } \{ W [ T ^ { k _ { 2 } } ( x _ { 2 j } x _ { 2 j } ^ { \prime } ) ] \} \sum _ { W ( y ) } \left\{\sum_{\Omega} Q_{1}\left\{W\left[T^{k_{1}}\left(x_{1 i} x_{1 i}^{\prime}\right)\right]\right\}\right.\right. \\
& \left.\left.\cdot P\left(W(y) \mid W\left[T^{k_{1}}\left(x_{1 i} x_{1 i}^{\prime}\right)\right], W\left[T^{k_{2}}\left(x_{2 j} x_{2 j}^{\prime}\right)\right]\right)\left[\sum_{\Omega} Q_{1}\left[W\left(x_{1 i^{*}} x_{1 i^{*}}^{\prime}\right)\right] Q_{2}\left[W\left(x_{2 j} x_{2 j}^{\prime}\right)\right] L^{s}\left(i^{*}, i\right)\right]^{\rho_{1}}\right\}\right] .
\end{aligned}
$$


decoding the second sender's message correctly satisfies

$$
\begin{aligned}
\bar{P}_{1} \leq & \bar{P}_{1,1}+\bar{P}_{1,2} \\
\leq & \exp \left\{-(\beta n-d)\left[E_{1}\left(\rho_{1}, q, p\right)-\rho_{1} R_{1}-\frac{2 \ln (d)}{\beta n-d}\right]\right\} \\
& +\exp \{-[(1-\beta) n-d] \\
& \left.\cdot\left[E_{1}\left(\rho_{1}^{\prime}, q^{\prime}, p\right)-\rho_{1}^{\prime} R^{\prime}-\frac{2 \ln (d)}{(1-\beta) n-d}\right]\right\}
\end{aligned}
$$

Proceeding in exactly the same manner, we obtain similar upper bounds for the probabilities, $\bar{P}_{2}$ and $\bar{P}_{3}$, of decoding errors in the second sender's message alone and in both messages, respectively. We conclude that the average error probability on the asynchronous two-sender channel satisfies

$$
\begin{aligned}
\bar{P}_{e, \text { async }} \leq & \sum_{\alpha=1}^{3}[\exp \{-(\beta n-d) \\
& \left.\cdot\left[E_{\alpha}\left(\rho_{\alpha}, q, p\right)-\rho_{\alpha} R_{\alpha}-\frac{2 \ln (d)}{\beta n-d}\right]\right\} \\
& +\exp \{-[(1-\beta) n-d] \\
& \left.\left.\cdot\left[E_{\alpha}\left(\rho_{\alpha}^{\prime}, q^{\prime}, p\right)-\rho_{\alpha}^{\prime} R_{\alpha}^{\prime}-\frac{2 \ln (d)}{(1-\beta) n-d}\right]\right\}\right],
\end{aligned}
$$

where $\rho_{\alpha}$ and $\rho_{\alpha}^{\prime}$ are between or equal to zero and one for $\alpha=1,2$, and 3. This expression for the asynchronous channel is analogous to (1) for the synchronous channel.

To obtain the cutoff rate region for the asynchronous channel, the exponents in (20) must be maximized with respect to $\rho_{\alpha}$ and $\rho_{\alpha}{ }^{\prime}$ for $\alpha=1,2$, and 3. Just as for the synchronous channel, the maximum with respect to $\rho_{\alpha}$ and $\rho_{\alpha}^{\prime}$ occurs at $\rho_{\alpha}=1$ and $\rho_{\alpha}^{\prime}=1$ for $\alpha=1,2$, and 3 . Therefore, the cutoff rate region for the asynchronous channel is the convex hull of the union, over all independent probability distributions $q(u, v)=q_{1}(u) q_{2}(v)$, of the set of all rate pairs $\left(R_{1}, R_{2}\right)$ satisfying

$$
\begin{array}{r}
R_{1} \leq-\ln \left(\sum_{Y} \sum_{X_{2}} q_{2}(v)\left[\sum_{X_{1}} q_{1}(u) p^{1 / 2}(w \mid u, v)\right]^{2}\right) \\
-(2 \ln (d))[\beta n-d]^{-1} \\
R_{2} \leq-\ln \left(\sum_{Y} \sum_{X_{1}} q_{1}(u)\left[\sum_{X_{2}} q_{2}(v) p^{1 / 2}(w \mid u, v)\right]^{2}\right) \\
-(2 \ln (d))[\beta n-d]^{-1}
\end{array}
$$

and

$$
\begin{array}{r}
R_{1}+R_{2} \leq-\ln \left(\sum_{Y}\left[\sum_{X_{1}} \sum_{X_{2}} q(u, v) p^{1 / 2}(w \mid u, v)\right]^{2}\right) \\
-(2 \ln (d))[\beta n-d]^{-1} .
\end{array}
$$

Since $\ln (d)[\beta n-d]^{-1}$ tends to zero as $n$ tends to infinity, the cutoff rate region defined by (21)-(23) for an asynchronous channel is the same as that defined by (2)-(4) for a synchronous channel. We note that the rate pair for the code is $\left(R_{1}^{*}, R_{2}^{*}\right)=\beta\left(R_{1}, R_{2}\right)+(1-$ $\beta)\left(R_{1}^{\prime}, R_{2}^{\prime}\right)$ and the average probability of error, $\bar{P}_{e \text {, async, }}$ tends to zero as $n$ tends to infinity.

A comparison of (1) and (20) indicates, however, that the average error probability for an asynchronous channel is potentially greater than that for a synchronous channel for the same block length and sender rates. Hence, to achieve a specified level of performance, one needs to select a larger block length for an asynchronous channel in comparison to a synchronous channel. For a given rate pair $\left(R_{1}, R_{2}\right)$, conservative estimates of the block lengths $n_{\text {sync }}$ and $n_{\text {async }}$ for the synchronous and asynchronous channels, respectively, required to achieve a specified level of performance can be determined as seen in the following examples.

Example 2 (Binary Adder Channel): Suppose for the channel of Example 1 that the operating rate pair is (0.6, $0.6), d=10$, and the block error probability is to be less than $10^{-5}$. Then the block lengths $n_{\text {sync }}=83$ and $n_{\text {async }}=$ 123 assure block codes exist that achieve the desired performance. About a 50 percent increase in complexity as measured by the block length is needed for equivalent performance.

Example 3 (Additive White Gaussian Noise Channel): Suppose the received signal is $r(t)=s_{1}(t ; i)+s_{2}(t ; j)+$ $w(t)$, where $s_{1}(t ; i)$ is the signal of sender- 1 for his $i$ th code symbol, $s_{2}(t ; j)$ is the signal of sender- 2 for his $j$ th code symbol, and $w(t)$ is a white Gaussian noise. Assume that each sender has a binary code alphabet, that the signal energy per bit to noise power density ratio is two for each sender, and that the demodulator alphabet dimension is infinite (i.e., there is no output quantization). If the signal sets of the two senders form mutually orthogonal simplexes, we find the cutoff rate region to be given by the set of rate pairs $\left(R_{1}, R_{2}\right)$ that satisfy $R_{1} \leq 0.68, R_{2} \leq 0.68$, and $R_{1}+R_{2} \leq 1.36$ bits. When operating with rate pair $(0.5,0.5), d=10$, and a required block error probability of $10^{-5}$, the estimated block lengths needed to assure the existence of a code are $n_{\text {sync }}=102$ and $n_{\text {async }}=149$, which again represents about a 50 percent increase in complexity for equivalent performance.

\section{Capacity Region Without Frame SYNCHRONIZATION}

The conclusion of Cover, McEliece, and Posner [1] that the capacity region remains unaffected in the absence of synchronization can be seen in an alternative manner from 
(20). From Gallager [4], it is evident that, for $\alpha=1,2$, and 3 , evaluating

$$
\partial\left[E_{\alpha}\left(\rho_{\alpha}, q, p\right)-\rho_{\alpha} R_{\alpha}-\frac{2 \ln (d)}{\beta n-d}\right] / \partial \rho_{\alpha}
$$

for $\rho_{\alpha}=0$, and noting that the result is nonnegative, yields a region of rate pairs whose convex closure is the capacity region. On doing this, we obtain the capacity region for an asynchronous channel as the convex hull of the union, over all independent probability distributions $q(u, v)=$ $q_{1}(u) q_{2}(v)$, of the set of all rate pairs $\left(R_{1}, R_{2}\right)$ that satisfy

$$
\begin{aligned}
& R_{1} \leq \partial E_{1}(0, q, p) / \partial \rho_{1}=I(u ; w \mid v) \\
& R_{2} \leq \partial E_{2}(0, q, p) / \partial \rho_{2}=I(v ; w \mid u)
\end{aligned}
$$

and

$$
R_{1}+R_{2} \leq \partial E_{3}(0, q, p) / \partial \rho_{3}=I(u, v ; w) .
$$

This is identical to the capacity region of a synchronous channel.

\section{Conclusion}

Cover, McEliece, and Posner have established the interesting and important result that the capacity region for a two-sender memoryless discrete channel is the same with and without synchronization for block coding. We have shown that the cutoff rate region, which is a subregion of the capacity region, is likewise the same with and without synchronization. We feel this is of potential importance because of the way the cutoff rate for a single user channel has come to play an important role in system design.

\section{ACKNOWLEDGMENT}

We are grateful to Professor James Massey for several helpful suggestions.

\section{REFERENCES}

[1] T. M. Cover, R. J. McEliece, and E. C. Posner, "Asynchronous multiple-access channel capacity," IEEE Trans. Inform. Theory, vol. IT-27, no. 4, pp. 409-413, July 1981.

[2] J. M. Wozencraft and R. S. Kennedy, "Modulation and demodulation for probabilistic coding," IEEE Trans. Inform. Theory, vol. IT-12, no. 3, July 1966.

[3] J. L. Massey, "Coding and modulation in digital communications," Proc. Int. Zurich Seminar on Digital Communications, Zurich, Switzerland, Mar. 1974.

[4] R. G. Gallager, Information Theory and Reliable Communcation. New York: Wiley, 1968.

[5] A. J. Viterbi, "Error bounds for convolutional codes and an asymptotically optimum decoding algorithm," IEEE Trans. Inform. Theory, vol. IT-13, pp. 260-269, Apr. 1967.

[6] D. L. Snyder and I. B. Rhodes, "Some implications of the cutoff rate criterion for coded direct detection optical communication systems," IEEE Trans. Inform. Theory, vol. IT-26, no. 3, pp. 327-338, May 1980.

[7] L. N. Lee, "On optimal soft decision demodulation," IEEE Trans. Inform. Theory, vol. IT-22, no. 4, July 1976.

[8] A. J. Viterbi, "Spread spectrum communications-myths and realities," IEEE Commun. Soc. Magazine, vol. 17, no. 3, pp. 11-18, May 1979.

[9] V. W. S. Chan, "A multiple user random access optical communication system," Proc. 1979 Int. Commun. Conf., Washington, DC, pp. 1.4.1-1.4.5, 1979 .

[10] E. A. Bucher, "Coding options for efficient communication on nonstationary channels," Proc. $1980 \mathrm{Int}$. Commun. Conf., Seattle, WA, pp. 4.1.1-4.1.7, June 1980.

[11] D. Slepian and J. K. Wolf, "A coding theorem for multiple access channels," Bell System Tech. J., vol. 52, pp. 1037-1076, 1973.

[12] N. T. Gaarder and J. K. Wolf, "The capacity region of a multiple access discrete memoryless channel can increase with feedback," IEEE Trans. Inform. Theory, vol. IT-21, pp. 100-102, Jan. 1975. 